\title{
PLC and SCADA Based Control of Continuous Stirred Tank Reactor (CSTR)
}

\author{
Mohd. Abdul Muqeet ${ }^{1}$, M.A.Najeeb ${ }^{2}$, Mrs. Fabia Akbar ${ }^{3}$, Sidra Ali $^{4}$ \\ Associate Professor, Dept. of Electrical Engineering, MJCET, Hyderabad, India ${ }^{1}$ \\ Lecturer Dept. of Electronics and Telecommunication Engineering, MPVPI, Nanded, India ${ }^{2}$ \\ Associate Professor, Dept. of Electrical Engineering, MJCET, Hyderabad, India ${ }^{3}$ \\ UG Student, Dept. of Electrical Engineering, MJCET, Hyderabad, India ${ }^{4}$
}

\begin{abstract}
In this paper we proposed to develop an experimental set-up of PLC and SCADA based mixing and control of Continuous Stirred Tank Reactor (CSTR). The set-up automatically monitors different parameters with the help of SCADA HMI. The PLC is connected to the level sensors and solenoid valves are controlled based on the level in the tank reactor. This model also has the stirrer control unit which continuously stirrers the reactor and mix the reactants. The PLC used is Allen Bradley Micrologix 1200 and the SCADA software is RSView32. Thus the level in the CSTR is constantly monitored and brought to a constant level as required for the operation. The automation is further enhanced by constant monitoring using SCADA screen which is connected to the PLC by means of RS232 communication cable.
\end{abstract}

Index Terms: Automation PLC, SCADA, CSTR, Level Control.

\section{INTRODUCTION}

Automation is the use of control systems and information technologies to reduce the need for human work in the production of goods and services.

The main advantages of automation are:

- Replacing human operators in tasks that involve hard physical or monotonous work.

- Replacing humans in tasks done in dangerous environments (i.e. fire, space, volcanoes, nuclear facilities, underwater, etc.)

- Performing tasks that are beyond human capabilities of size, weight, speed, endurance, etc.

- Economy improvement: Automation may improve in economy of enterprises, society or most of humanity.

Engineers now can have numerical control over automated devices. Information technology, together with industrial machinery and processes, can assist in the design, implementation, and monitoring of control systems [1]. One example of an industrial control system is a programmable logic controller (PLC). PLCs are specialized hardened computers which are frequently used to synchronize the flow of inputs from (physical) sensors and events with the flow of outputs to actuators and events. PLCs may need to interact with people for the purpose of configuration, alarm reporting or everyday control [8]. A Human-machine interfaces (HMI) or computer human interfaces $(\mathrm{CHI})$, formerly known as man-machine interfaces, and graphical user interface (GUIs) are usually employed to communicate with PLCs and other computers [3]. Most modern PLCs can communicate over a network to some other system, such as a computer running a SCADA (Supervisory Control and Data Acquisition) system or web browser. PLCs used in larger $\mathrm{I} / \mathrm{O}$ systems may have peer-to-peer (P2P) communication between processors. This allows separate parts of a complex process to have individual control while allowing the subsystems to co-ordinate over the communication link [3].

The industrial application of liquid level control is tremendous especially in refineries petroleum and chemical process industries. Usually, level control exists in some of the control loops of a process control system [2].

A continuous stirred tank reactor is one example in which a liquid level control system is a part of control loop. CSTRs are used in many process industries for the purpose of mixing of two or more reactants [1].

In a CSTR, reactants are fed to the reactor at the inlet and the mixed product is removed from the reactor at the outlet. Level control is also very important for mixing reactant process. The quality of the product of the mixture depends on the level of the reactants in the mixing CSTR. Mixing reactant process is a very common process in chemical process industries and food processing industries Many other industrial applications are concerned with level control, may it be a single loop level control or sometimes multi-loop level control [4]. In our proposed work we develop PLC and SCADA based experimental set-up of a Continuous stirred tank reactor (CSTR). Section II describes the hardware components used in this work. Section III provides the software description and different software tools used in this work. Implementation details are given in Section IV. Results are depicted in Section V and conclusion is provided in Section VI.

\section{HARDWARE DESCRIPTION}

Fig. 1 represents the general block diagram of our experimental set-up. The main objective of the set-up is to provide a real time implementation of PLC and SCADA based level control of CSTR. As shown in the block diagram PLC can take input from various sensors or 
transmitters. Control valves can be controlled by the programming of PLC which are connected to the outputs of the PLC. Stirrer motor and pumps are also controlled by the programming of PLC. Continuous monitoring is possible by using SCADA unit.

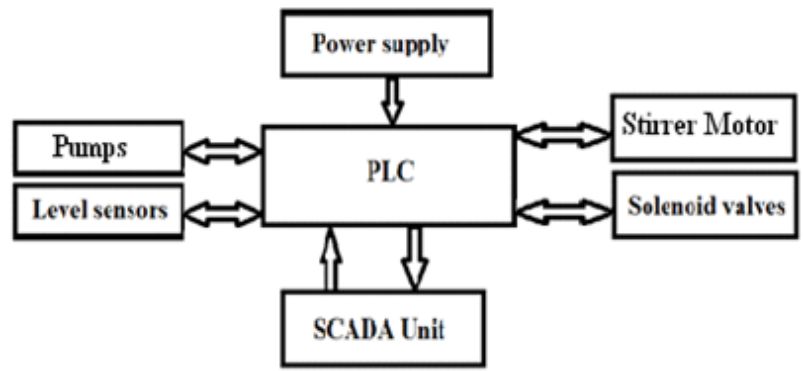

Fig.1 General Block Diagram of the Experimental Set-up

A schematic representation of the experimental set-up is also shown in the Fig.2.As per the block diagram and schematic diagram; the main hardware components used in this experimental set-up are listed below:

- Power supply unit (switched mode power supply):

- Allen Bradley PLC Micrologix 1200 (Modular Type)

- RS-232 cable to communicate with SCADA unit.

- Pumps (3 Nos.)

- Solenoid Valves (3 Nos.)

- Stirrer and motor unit

- Tanks(Two tanks for storing the reactants and one main big for CSTR)

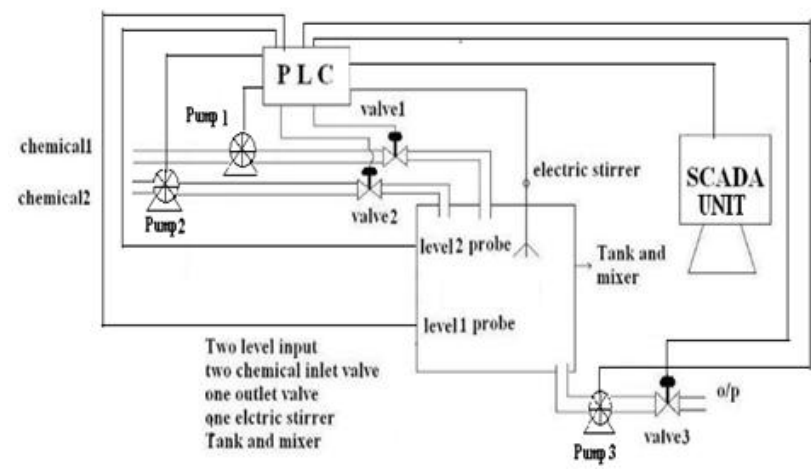

Fig.2 Schematic Diagram of the CSTR

The Power supply unit is a portable power supply of $2.5 \mathrm{~A} / 24 \mathrm{~V}$ DC. The pump has current capacity of $0.23 \mathrm{~A}$ and Power rating of 18 watts. The tanks used for the experimental setup are of transparent plastic material of high impact chemical resistance. For storing the reactants tank capacity is 1 litres and the capacity of the main CSTR tank is 3 litres. For the stirrer motor control unit a DC motor of 100RPM, 12V DC is used. The stirrer used is plastic material having 4 blades with each blade diameter is 2 inch. We used DC motor for the stirrer unit with rating of $100 \mathrm{RPM} / 12 \mathrm{~V} \mathrm{DC}$. The torque is $1.2 \mathrm{Kgcm}$ with $6 \mathrm{~mm}$ diameter shaft with internal hole in it. Solenoid valves which are important component in our experimental set up are of size $1 / 2$ inch with voltage rating of $12 \mathrm{VDC}$ and current rating of $500 \mathrm{~mA}$. These solenoid valves with normally closed operation are selected to withstand the temperature upto $70^{\circ} \mathrm{C}$ and pressure upto 120 psi. For the
CSTR process we selected the ON/OFF type of magnetic float level sensors which gives either 24VDC or 0VDC. The idea behind this level sensor is that when there is no water in the tank contact is open and signal strength is $0 \mathrm{~V}$ DC and if sensor is immersed in the water contact is closed and the signal strength is $24 \mathrm{~V}$ DC. The PLC used is Allen Bradley Micrologix 1200. PLC is modular type with RS232 communication protocol. The software used to communicate the PLC with SCADA is RSLinx Classic ${ }^{\mathrm{TM}}$ and the programming platform for ladder programming the PLC is RSLogix500 [9], [10].

\section{SOFTWARE DESCRIPTION}

The software tools used for the experimental set-up are mainly for writing the PLC ladder program and preparing the GUI or screens of SCADA. The PLC used is Allen Bradley Micrologix 1200. The PLC is modular type with RS232 communication protocol. The software used to communicate the PLC with SCADA is RSLinx Classic ${ }^{\mathrm{TM}}$ [9] and the programming platform for programming the PLC is RSLogix500. RSLinx Classic ${ }^{\mathrm{TM}}$ is the most widely installed communication server in automation today. RSLinx Classic provides plant-floor device connectivity for a wide variety of Rockwell Software applications such as RSLogix ${ }^{\mathrm{TM}} 5 / 500 / 5000$ and RSView32 [9].

The RSLogix500 software is used to program and control Allen-Bradley PLC's with a PC-type computer. This software allows to create, edit, and monitor PLC ladder programs. It also allows to document ladder programs, to store projects (ladder program files and all other associated files) on disk, and to print complete reports on a project [10].

RSView32 is Windows-based software for developing and running human machine interface applications designed for use in Microsoft Windows NT and Windows9x [9]. RSView32 gives you all the tools needed to create and run effective monitoring and supervisory control applications. RSView32 Works contains both development and run time software [10]. Use this software to develop and run RSView32 applications.RSView32 Run time contains only run time software. Use this software to run applications developed in RSView32 Works [9]. A typical SCADA system comprises of $\mathrm{I} / \mathrm{O}$ signal hardware, controllers, software, networks and communication. SCADA system is normally used to monitor and control a remote field or a distribution that is spread out for a long distance [3].PLC are used to control the field operations and communicate the same to SCADA. The SCADA system also provides a host control functions for the supervisor to control and define settings [4].

\section{IMPLEMENTATION}

As per the schematic shown in Fig. 2 we are using the CSTR process in Non-Interacting mode where two tanks are used for storing the reactants and the main CSTR is used for mixing these two reactants. The two solenoid valves are used to control the flow from these two tanks into the main CSTR. These two solenoid valves acts as the input valves to the main CSTR. The solenoid valve connected at the outflow of main CSTR is used to discharge the mixed product. The level probes and the 
solenoid valves are connected to the PLC. The PLC is Pump1 is OFF and the valve2 and Pump2 are ON. When connected to the SCADA unit with RS232 cable. Upon the level of the CSTR is full as detected by the level probe initialization from the SCADA unit the PLC works according to the ladder programming.

the valve 2 and pump2 are made OFF and the stirrer motor The working of the set-up is explained here with reference to the ladder diagram [6], [7]. We are assuming that the upper two tanks are already filled as part of a different starts/ON the mixing operation of the two reactants set for a predefined time as programmed in the Ladder program. process. As soon as the master switch is switched ON the CSTR. This process is repeated as level in the CSTR process works as per the ladder program shown in Fig. 3, reaches to empty level indication. The SCADA software Fig.4 and Fig.5. When the main CSTR is empty as RSView32 Run Time continuously monitors the process detected by the level probes the PLC starts/ON the valve1 and same is depicted in the SCADA screen with the and Pump1. When the CSTR is half fill the valve1 and process mimic.

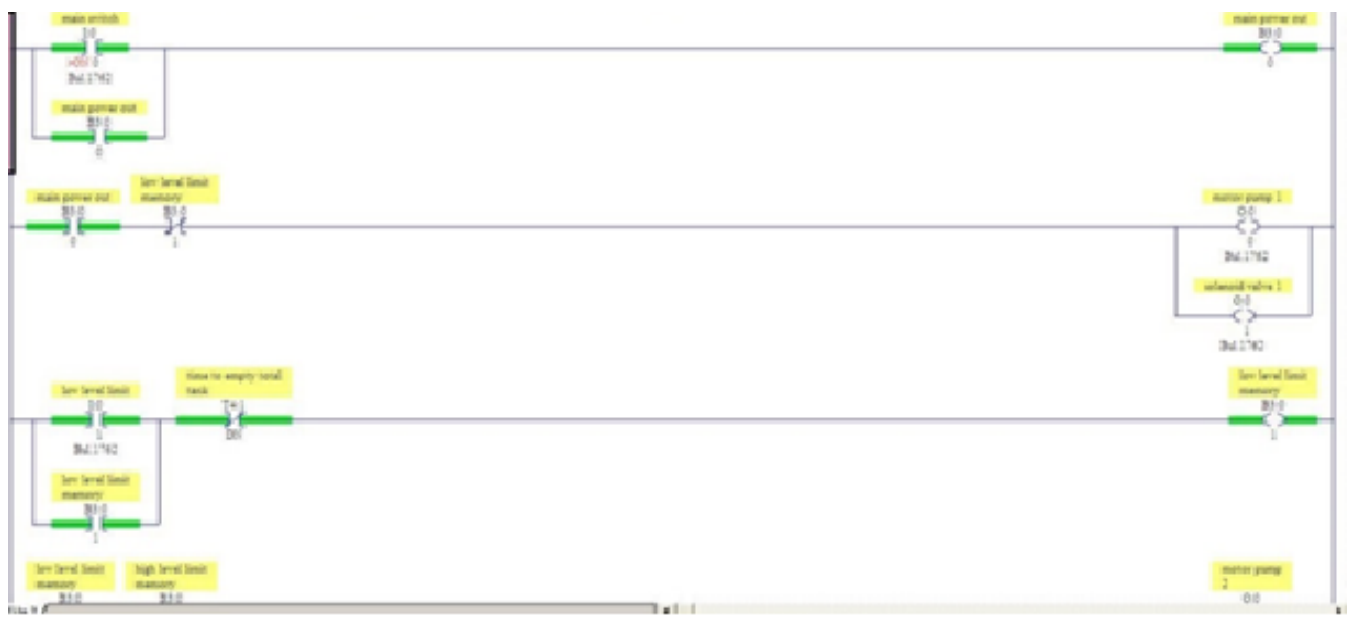

Fig 3.Ladder Program Part.1

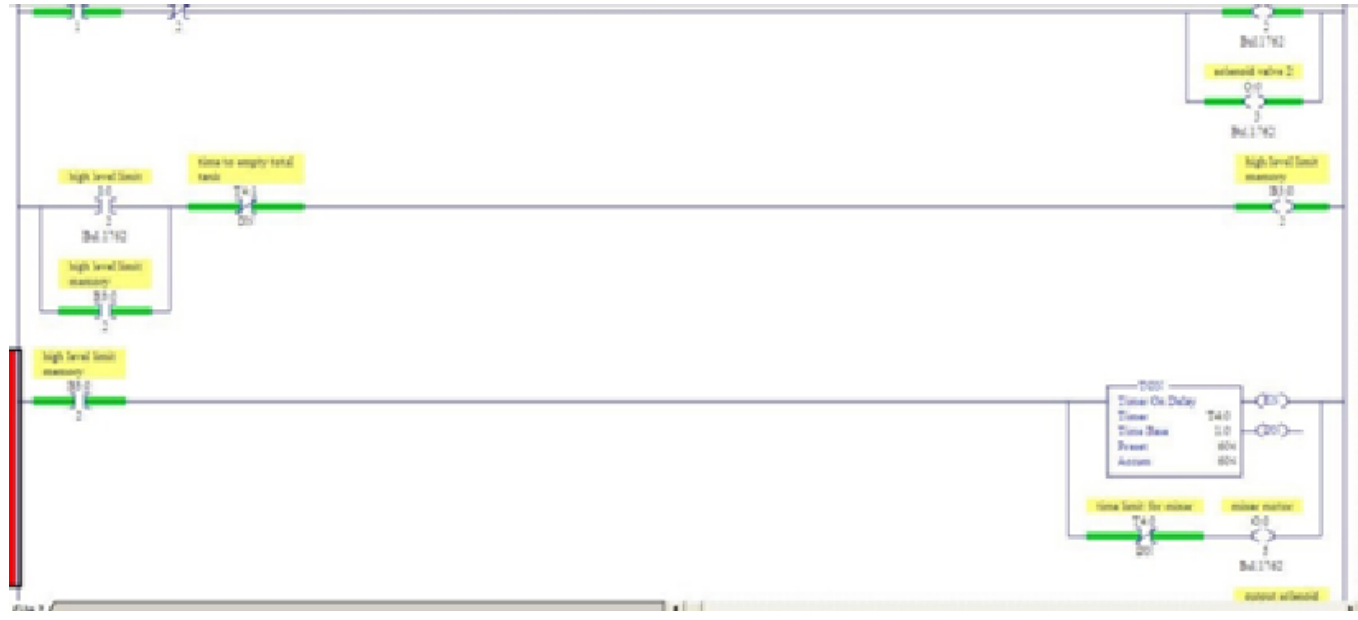

Fig 4.Ladder Program Part.2

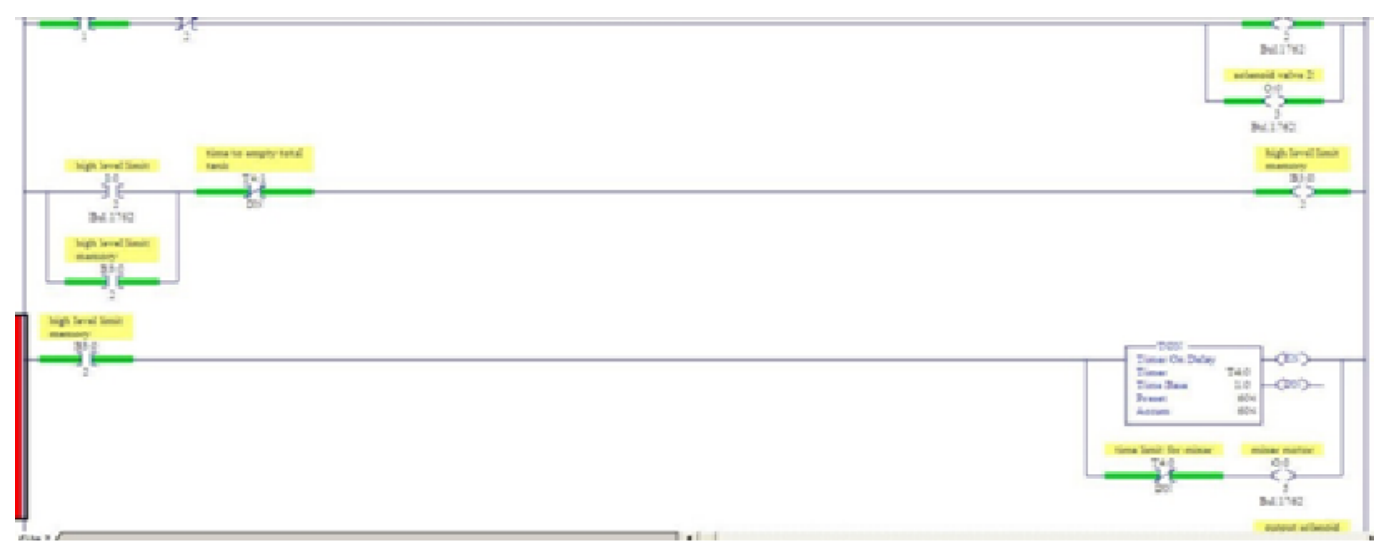

Fig 5.Ladder Program Part.3 
INTERNATIONAL JOURNAL OF INNOVATIVE RESEARCH IN ELECTRICAL, ELECTRONICS, INSTRUMENTATION AND CONTROL ENGINEERING Vol. 3, Issue 12, December 2015

\section{V.RESULTS}

The main objective of our paper is accomplished by designing the PLC and SCADA based mixing and control of CSTR. The PLC programming is done using RSLogix500 and entire process is monitored with RSView32 SCADA Run Time software. The main RSView32 SCADA screen is shown in Fig.6 which is developed with the help of RSView32 Works development environment and monitoring of the process is carried out in RSView32 Run Time environment. To prepare the SCADA screens we used RSView32 Works development environment which contains editors for creating a complete human-machine interface application. After preparing the SCADA screens we can switch to run mode or use RSView32 Runtime (which is included with RSView32 Works and uses less memory), and run our application. In the tag database, we defined the different variable data for RSView32 $2^{\mathrm{TM}}$ to monitor. The current value of a tag, when required, is updated from the device it is connected to and stored in computer memory-referred to as the value table - so it is immediately accessible to all parts of RSView32 [9].

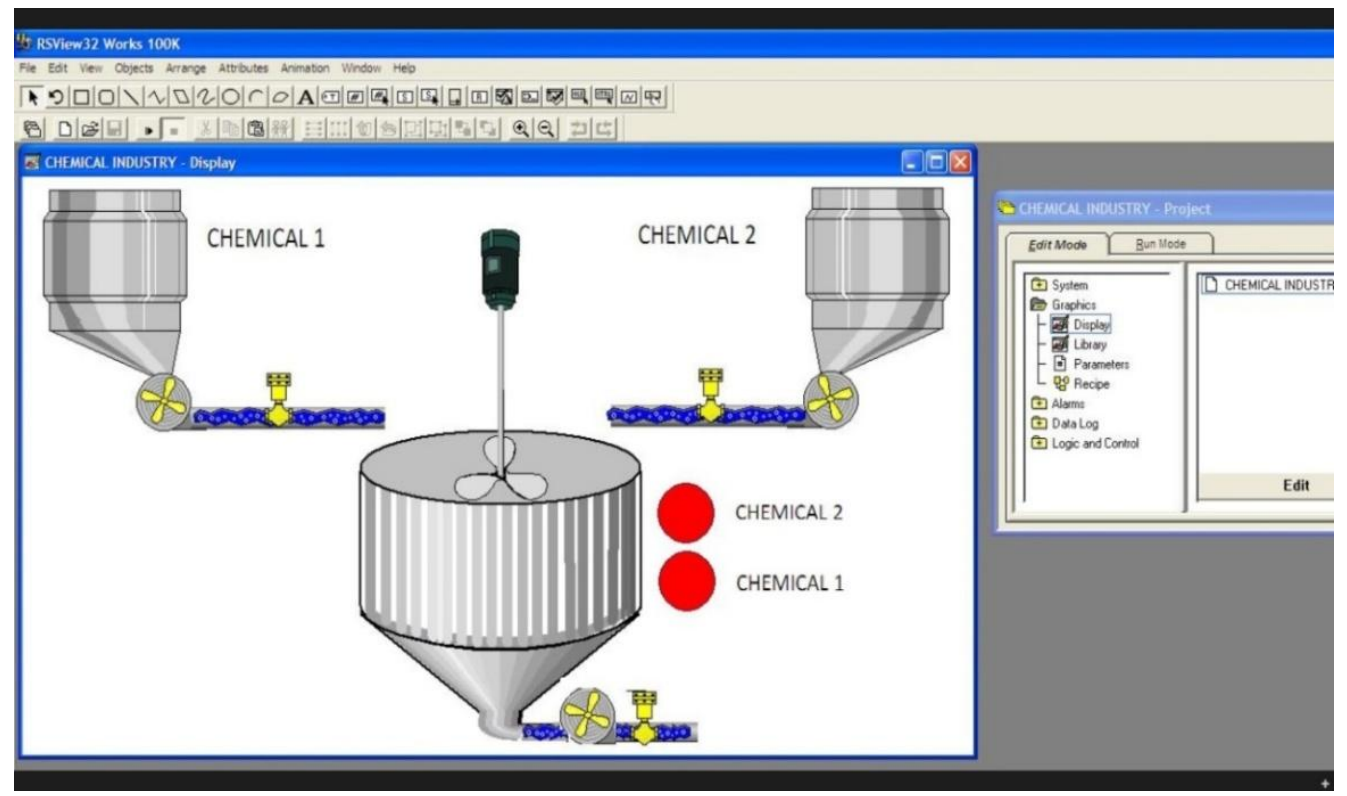

Fig.6 Main SCADA screen in RSView32 Run Time Environment

The actual hardware experimental set-up is shown in Fig.7.

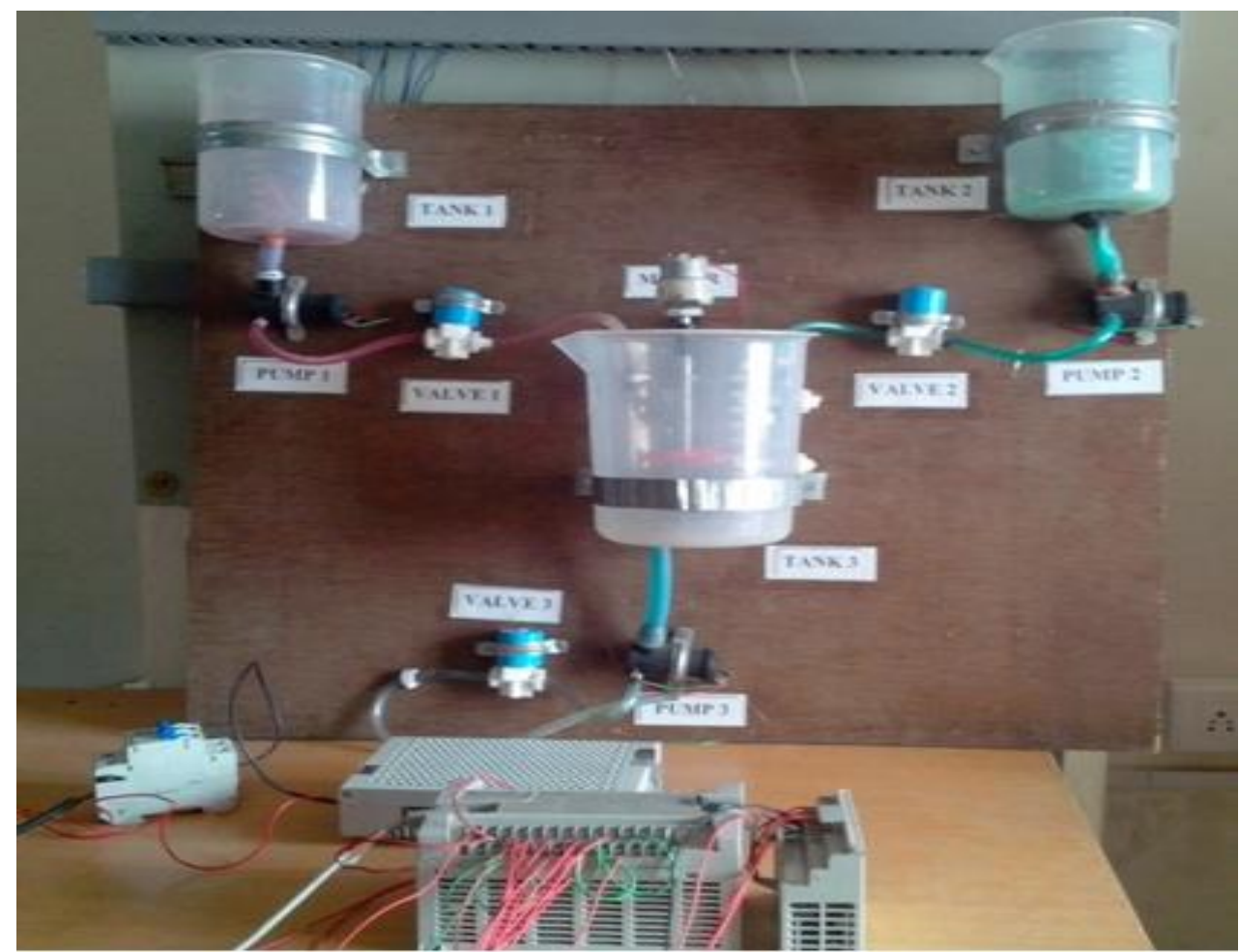

Fig.7 Hardware Experimental Set-up 


\section{CONCLUSION}

Thus we intend to provide an experimental set-up for PLC and SCADA based mixing and control of CSTR . This setup aims at developing a low cost effective automated system which may provide effective monitoring of the process. We can view, generate report, plots, alarm reporting as configured on the SCADA directly on the workstation/server or any other PC connected in the same network.

The following modification may be possible in the existing set-up:

1. Instead of RS232 communication we can have wireless communication with the SCADA server.

2. The ON/OFF type level sensors can be replaced with continuous signaling level sensors for more accurate level control of the reactor.

3. A PID based control ladder program can be written for controlling of level with continuous level sensors.

4. Instead of solenoid valves we can connect globe type valves for better inflow and outflow from the reactor

\section{ACKNOWLEDGMENT}

We like to acknowledge the effort of all the sources which were incorporated in our paper. We would also like to acknowledge the heartfelt effort given by our Director Dr. Basheer Ahmed sir, Dean of Academics Dr. Ashfaque Jafri sir and our HOD Dr. M.P. Soni sir for their valuable support.

\section{REFERENCES}

[1] Edward W. Kamen Industrial Controls and Manufacturing, (Academic Press, 1999) ISBN 0123948509.

[2] Artificial Intelligence Based Liquid Level Control of Coupled Tanks using Fuzzy Logic Controller, International Journal of Latest Trends in Engineering and Technology (IJLTET), Vol. 5 Issue 2 March 2015.

[3] Boyer, Stuart A. SCADA Supervisory Control and Data Acquisition, USA: ISA. p. 179. ISBN 978-1-936007-09-7.

[4] S.Kalaivani, M.Jagadeeswari, PLC \& SCADA Based Effective Boiler Automation System for Thermal Power Plant, International Journal of Advanced Research in Computer Engineering \& Technology (IJARCET), Volume 4 Issue 4, April 2015

[5] Rockwell Automation SCADA System Selection guide AllenBradley, Publication AG-2.1. 1998.

[6] Product Manual, MicroLogix TM 1200 Programmable Controllers, Rockwell Automation, Allen- Bradley, pp.39-73, 1999

[7] Frank D.Petruzella, Programmable Logic Controllers , 3rd edition, McGraw Hill, pages:20-32, 2010

[8] Rishabh Das, Sayantan Dutta, Anusree Sarkar,Kaushik Samanta, Automation of Tank Level Using PLC and Establishment of HMI by SCADA, IOSR-JEEE, Volume 7, Issue 2 (Jul. - Aug. 2013)

[9] http://literature.rockwellautomation.com/idc/groups/literature/docu ments/um/vw32-um001_-en-e.pdf

[10] http://www.engineer-and-technician.com/wpcontent/uploads/2013 /12/How-To-Program-RSView32-excerpt.pdf 